\title{
Changes in Direct Healthcare Costs before and after the Diagnosis of Inflammatory Bowel Disease: A Nationwide Population-Based Study
}

\author{
Jooyoung Lee ${ }^{1,2}$, Jong Pil Im¹, Kyungdo Han ${ }^{3}$, Jihye Kim ${ }^{1,4}$, Hyun Jung Lee ${ }^{1}$, Jaeyoung Chun ${ }^{1}$, and Joo Sung Kim ${ }^{1,2}$ \\ ${ }^{1}$ Department of Internal Medicine and Liver Research Institute, Seoul National University College of Medicine, ${ }^{2}$ Department of Internal \\ Medicine, Healthcare Research Institute, Seoul National University Hospital Healthcare System Gangnam Center, ${ }^{3}$ Department of Medical \\ Statistics, College of Medicine, Catholic University of Korea, and ${ }^{4}$ Department of Internal Medicine, CHA Gangnam Medical Center, CHA \\ University School of Medicine, Seoul, Korea
}

Background/Aims: We aimed to investigate the differences in direct healthcare costs between patients with and without inflammatory bowel disease (IBD) and changes in direct healthcare costs before and after IBD diagnosis. Methods: This population-based study identified 34,167 patients with IBD (11,014 patients with Crohn's disease and 23,153 patients with ulcerative colitis) and 102,501 age-and sexmatched subjects without IBD (the control group) from the National Health Insurance database using the International Classification of Disease, 10th revision codes and the rare intractable disease registration program codes. The mean healthcare costs per patient were analyzed for 3 years before and after IBD diagnosis, with follow-up data available until 2015. Results: Total direct healthcare costs increased and peaked at $\$ 2,396$ during the first year after IBD diagnosis, but subsequently dropped sharply to $\$ 1,478$ during the second year after diagnosis. Total healthcare costs were higher for the IBD patients than for the control group, even in the third year before the diagnosis ( $\$ 497$ vs $\$ 402$, $p<0.001$ ). The costs for biologics for the treatment of IBD increased steeply over time, rising from $\$ 720.8$ in the first year after diagnosis to $\$ 1,249.6$ in the third year after diagnosis $(p<0.001)$. Conclusions: IBD patients incurred the highest direct healthcare costs during the first year after diagnosis. IBD patients had higher costs than the control group even before diagnosis. The cost of biologics increased steeply over time, and it can be assumed that biologics could be the main driver of costs during the early period after IBD diagnosis. (Gut Liver 2020;14:89-99)

Key Words: Inflammatory bowel disease; Cost and cost analysis; Biologic product

\section{INTRODUCTION}

Inflammatory bowel disease (IBD) represented by Crohn's disease (CD) and ulcerative colitis (UC) can occur at any age but mostly affects young people. Although IBD has a low mortality rate, it is incurable and commonly requires lifelong care and medication. IBD inevitably generates a significant economic burden at the patient and societal levels. Several cost-of-illness studies have been performed in North America and Europe, which are regions with high incidence and prevalence of IBD. Although differences in data and methodologies created discrepancies among those studies, their findings confirmed that IBD was associated with a substantial economic burden. ${ }^{1-4}$

During the last two decades, the incidence and prevalence of IBD have risen in previously low-incidence areas, such as Asia, Eastern Europe, and many developing countries, while the incidence and prevalence of IBD have stabilized in North America and Europe. ${ }^{5,6}$ According to a series of studies that were conducted at different times, the incidence of IBD in South Korea has increased approximately 10-fold over the last two decades, which has led to South Korea having one of the highest incidences of IBD among Asian countries. ${ }^{7-10}$ Based on Western experience, the increases in the incidence and prevalence of IBD may lead to considerable economic challenges for the South Korean healthcare system. However, there is limited research regarding the healthcare costs of IBD in Asia. This study aimed to investigate the direct healthcare costs of IBD in South Korea, with a focus on the changes in the costs per patient before and after the IBD diagnosis.

\footnotetext{
Correspondence to: Jong Pil Im

Department of Internal Medicine and Liver Research Institute, Seoul National University College of Medicine, 101 Daehak-ro, Jongno-gu, Seoul 03080, Korea

Tel: +82-2-2072-0638, Fax: +82-2-742-8601, E-mail: jpim0911@snu.ac.kr Received on January 15, 2019. Revised on March 28, 2019. Accepted on April 17, 2019. Published online December $11,2019$. pISSN 1976-2283 eISSN 2005-1212 https://doi.org/10.5009/gnl19023

() This is an Open Access article distributed under the terms of the Creative Commons Attribution Non-Commercial License (http://creativecommons.org/licenses/by-nc/4.0) which permits unrestricted non-commercial use, distribution, and reproduction in any medium, provided the original work is properly cited.
} 


\section{MATERIALS AND METHODS}

\section{Data source}

This nationwide population-based study evaluated data from the National Health Insurance (NHI) database. The NHI is a non-profit single payer organization under the South Korean government responsible for the NHI program. The NHI system covers approximately 97\% of the South Korean population and provides comprehensive data regarding patient demographics, medical use/transaction information, other insurance coverage, patient co-pay fees, and case-specific claims (diagnosis, prescriptions, and consultation statements). ${ }^{11,12}$ In 2007, the NHI established a registration program for rare intractable disease (RID), which included IBD, to provide enhanced reimbursement for medical costs that were associated with rare disease (affecting $<20,000$ people in Korea). To qualify for enrollment in the RID program, patients with IBD had to fulfil the diagnostic criteria provided by NHI, based on clinical features, endoscopic findings, and histologic findings. The accuracy of the RID database for both CD and UC diagnosis have been validated through previous studies. $^{8,13,14}$

\section{Study population and patient identification}

We evaluated the data of patients with IBD who were identified in the NHI database between 2010 and 2012, using codes from the International Classification of Disease, 10th revision (ICD-10), and the RID registration system (V code). Cases that involved CD were identified if they had both ICD-10 code K50 and V code V130, while cases that involved UC were identified if they had both ICD-10 code K51 and V code V131. To verify the diagnostic accuracy of the IBD defined with the ICD10 code and V code, we reviewed the medical records of 830 patients with IBD who were registered at Seoul National University Hospital, a tertiary referral hospital in Korea from January 2010 to December 2013. The combination of these two codes provided 94.5\% (312/330) sensitivity for CD diagnosis and 96.4\% (482/500) sensitivity for UC diagnosis. Patients with IBD were subsequently divided into an "incident group" and "prevalent group." The incident group included all patients who were newly identified using both the ICD-10 and V codes for CD and UC from January 2010 to December 2012. These patients had no history of CD or UC from January 2005 until the inclusion date of this study. The prevalent group included all the patients who were formerly assigned IBD codes, from 2005 to the inclusion date, and received reimbursements for IBD during the 2010 to 2012 period. In addition, age- and sex-matched individuals without IBD (the control group) were randomly selected and included in the study at a 3:1 ratio of controls to case.

\section{Calculation of healthcare cost}

The direct healthcare costs of IBD were analyzed for 3 years before and after the IBD diagnosis, with follow-up data avail- able until 2015. All costs were estimated on the basis of claimed costs rather than the actual amounts that were paid by the patients, and the mean direct healthcare costs per patient were calculated. Although median costs are often used in cohort studies to avoid bias resulting from high costs that are incurred by a minority of patients, the mean costs are important for planning future healthcare budgets as they estimate the overall predicted expenditure. ${ }^{15-17}$ Costs were expressed in US dollars (\$), based on an exchange rate of 1,110 South Korean won per US dollars on November 1, 2017. Total IBD-related direct healthcare costs were categorized as inpatient and outpatient costs. Inpatient costs were defined as the sum of costs incurred during the hospital stay in either medical or surgical wards, which was related to the diagnosis or management of IBD. Outpatient costs were defined as the sum of costs for office visits, emergency department visits, endoscopic procedures, radiologic procedures, and other outpatient services. Cost of surgery included all IBDrelated surgery, from minor surgery such as abscess drainage to major surgery such as bowel resection. Costs of diagnostic test were calculated separately for endoscopy and radiologic imaging. Endoscopy included flexible sigmoidoscopy, colonoscopy, esophagogastroduodenoscopy, enteroscopy and capsule endoscopy, which were performed at the IBD diagnosis and throughout the follow-up period. Radiologic imaging included abdominal sonography, small bowel series, computerized tomography, and magnetic resonance imaging. Costs of IBD medication were only dealt with biologics (infliximab and adalimumab) imposed by the in-hospital prescription code. The prescription details and billing code of drugs with "in-hospital prescription" are more accurately recorded and collected in the NHI database, compared to that of the medication prescribed as an "out-of-hospital prescription." Comorbidities that could affect healthcare costs were adjusted for calculation and comparison of total direct healthcare cost. We used ICD-10 codes of all comorbidities that could be extracted from the NHI database to substitute them for the Charlson comorbidity index calculation, and scored and applied them to all subjects of this study.

\section{Statistical analysis}

All statistical analyses were performed using the R software version 3.4.3 (The R Foundation for Statistical Computing, Vienna, Austria, http://www.R-project.org) and SAS software version 9.3 (SAS institute, Cary, NC, USA). Random selection of ageand sex-matched controls was performed using the SAS algorithm. Continuous variables were reported as mean \pm standard deviation and categorical variables were reported as number and percentage. Multiple comparisons were used to compare the differences in costs between groups. Continuous variables were analyzed using the Student t-test, while binary and categorical variables were analyzed using the chi-square test. Differences were considered to be statistically significant at $\mathrm{p}$-value of $<0.05$. 


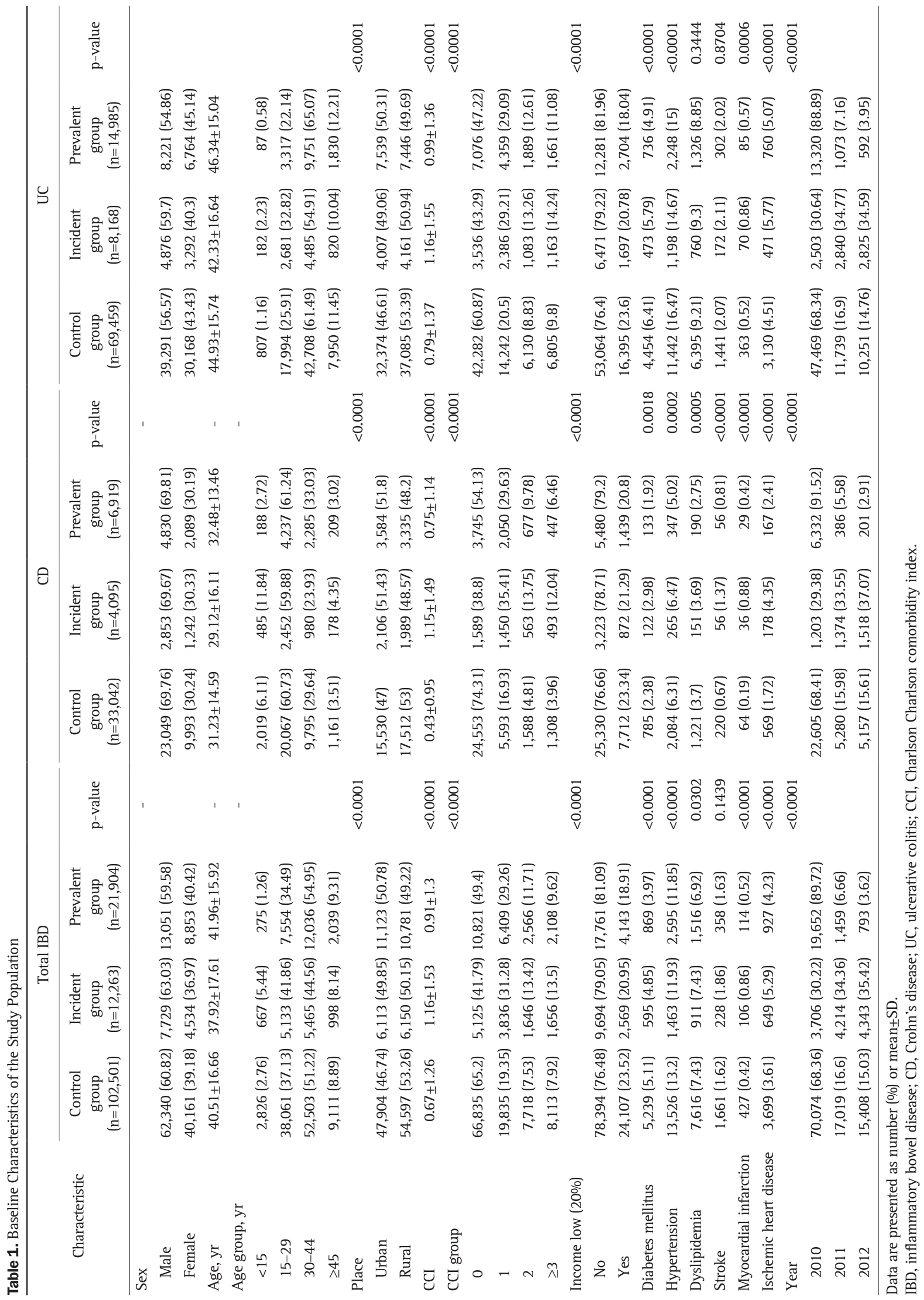


Table 2. Changes in Direct Healthcare Costs before and after the Diagnosis of IBD

\begin{tabular}{|c|c|c|c|c|c|c|}
\hline & Pre 3 yr & Pre 2 yr & Pre $1 \mathrm{yr}$ & $1 \mathrm{yr}$ & $2 \mathrm{yr}$ & $3 \mathrm{yr}$ \\
\hline \multicolumn{7}{|l|}{ Total healthcare cost, $\$$} \\
\hline \multicolumn{7}{|l|}{ Total IBD } \\
\hline Control group & 402 & 472 & 574 & 616 & 631 & 628 \\
\hline Prevalent group & 940 & 1,086 & 1,253 & 1,560 & 1,579 & 1,654 \\
\hline Incident group & 497 & 616 & 983 & 2,396 & 1,478 & 1,437 \\
\hline \multicolumn{7}{|l|}{$\mathrm{CD}$} \\
\hline Prevalent group & 1,399 & 1,670 & 2,030 & 2,659 & 2,714 & 2,843 \\
\hline Incident group & 442 & 567 & 1,225 & 3,658 & 2,109 & 2,120 \\
\hline \multicolumn{7}{|l|}{$\mathrm{UC}$} \\
\hline Prevalent group & 728 & 814 & 892 & 1,036 & 1,051 & 1,098 \\
\hline Incident group & 539 & 646 & 879 & 1,758 & 1,185 & 1,117 \\
\hline \multicolumn{7}{|l|}{ Diagnostic tests } \\
\hline \multicolumn{7}{|l|}{ Cost of endoscopy, \$ } \\
\hline Control group & 2.9 & 3.2 & 3.5 & 3.8 & 3.9 & 3.8 \\
\hline Prevalent group & 21.7 & 24.1 & 24.5 & 24.7 & 18.2 & 18.6 \\
\hline Incident group & 6.9 & 9.8 & 31.9 & 53.2 & 20.6 & 18.8 \\
\hline $\mathrm{CD}$ & 6.2 & 8.8 & 36.1 & 51.9 & 18.1 & 17.8 \\
\hline $\mathrm{UC}$ & 7.2 & 10.3 & 29.8 & 53.8 & 21.8 & 19.4 \\
\hline \multicolumn{7}{|c|}{ Cost of radiologic imaging, $\$$} \\
\hline Control group & 2.7 & 3.4 & 4.3 & 5.0 & 4.9 & 4.8 \\
\hline Prevalent group & 16.8 & 20.4 & 23.4 & 25.6 & 20.7 & 18.9 \\
\hline Incident group & 4.2 & 6.9 & 21.0 & 53.8 & 18.4 & 17.6 \\
\hline $\mathrm{CD}$ & 5.8 & 10.0 & 42.2 & 106.8 & 35.0 & 36.7 \\
\hline $\mathrm{UC}$ & 3.8 & 5.8 & 12.6 & 28.1 & 10.7 & 8.6 \\
\hline \multicolumn{7}{|l|}{ Cost of surgery, $\$$} \\
\hline Control group & 0.3 & 0.4 & 0.6 & 0.6 & 0.7 & 0.7 \\
\hline Prevalent group & 6.8 & 7.6 & 10.2 & 12.9 & 9.5 & 8.9 \\
\hline Incident group & 1.3 & 2.0 & 5.6 & 25.9 & 7.4 & 5.6 \\
\hline $\mathrm{CD}$ & 3.0 & 4.7 & 13.2 & 59.2 & 11.3 & 11.4 \\
\hline $\mathrm{UC}$ & 0.5 & 0.7 & 1.5 & 8.0 & 5.3 & 2.6 \\
\hline \multicolumn{7}{|l|}{ Cost of biologics, $\$$} \\
\hline \multicolumn{7}{|l|}{ Total IBD } \\
\hline Control group & 0.6 & 0.7 & 0.9 & 2.3 & 3.0 & 3.4 \\
\hline Prevalent group & 93.9 & 111.8 & 201.9 & 400.0 & 523.7 & 648.4 \\
\hline Incident group & 5.5 & 7.8 & 9.2 & 320.7 & 482.6 & 601.2 \\
\hline \multicolumn{7}{|l|}{$\mathrm{CD}$} \\
\hline Prevalent group & 281.7 & 338.6 & 610.6 & $1,134.7$ & 1,327.9 & $1,584.1$ \\
\hline Incident group & 11.1 & 13.9 & 19.3 & 774.4 & $1,052.4$ & $1,274.0$ \\
\hline \multicolumn{7}{|l|}{ UC } \\
\hline Prevalent group & 0.1 & 6.2 & 11.4 & 57.0 & 147.9 & 210.3 \\
\hline Incident group & 6.1 & 8.3 & 10.2 & 108.1 & 215.0 & 281.8 \\
\hline
\end{tabular}

Data are presented as mean.

IBD, inflammatory bowel disease; CD, Crohn's disease; UC, ulcerative colitis. 


\section{Ethical consideration}

All data were obtained from the NHI database in an encrypted and anonymous state; therefore, informed consent from the study participants was not required. This study's retrospective protocol was approved by the Institution Review Board of Seoul National University Hospital (IRB number: H-1703-107-840).

\section{RESULTS}

\section{Baseline characteristics of the study population}

We extracted the records of 23,153 patients who were diagnosed with UC and 11,014 patients who were diagnosed with $\mathrm{CD}$ from the NHI database during the study period (the mean age was $40.5 \pm 16.7$ years and $61.0 \%$ were male). Among the UC patients, 8,168 and 14,985 patients were classified as the incident group and the prevalent group, respectively. Meanwhile, among the CD patients, 4,095 patients and 6,919 patients were included in the incident group and the prevalent group, re- spectively. The control group comprised 102,501 age-and sexmatched subjects without IBD. The baseline characteristics of the study population are summarized in Table 1. Compared to the control group, the IBD group had significantly lower proportions of rural residents and individuals with lower 20\% incomes $(p<0.001)$. The IBD group had a significantly lower prevalence of diabetes mellitus, hypertension and dyslipidemia than the control group. However, IBD patients had higher prevalence of myocardial infarction and ischemic heart disease than individuals without IBD $(\mathrm{p}<0.001)$.

\section{Total healthcare costs}

The changes in total direct healthcare costs per patient in these two groups are depicted in Table 2. IBD group had higher total direct healthcare costs than the control group throughout the study period. Total healthcare costs per patient in the incident group were even higher compared to the costs of the control group from 3 years before the diagnosis $(\mathrm{p}<0.001)$.

In the incident group, the total healthcare costs peaked at
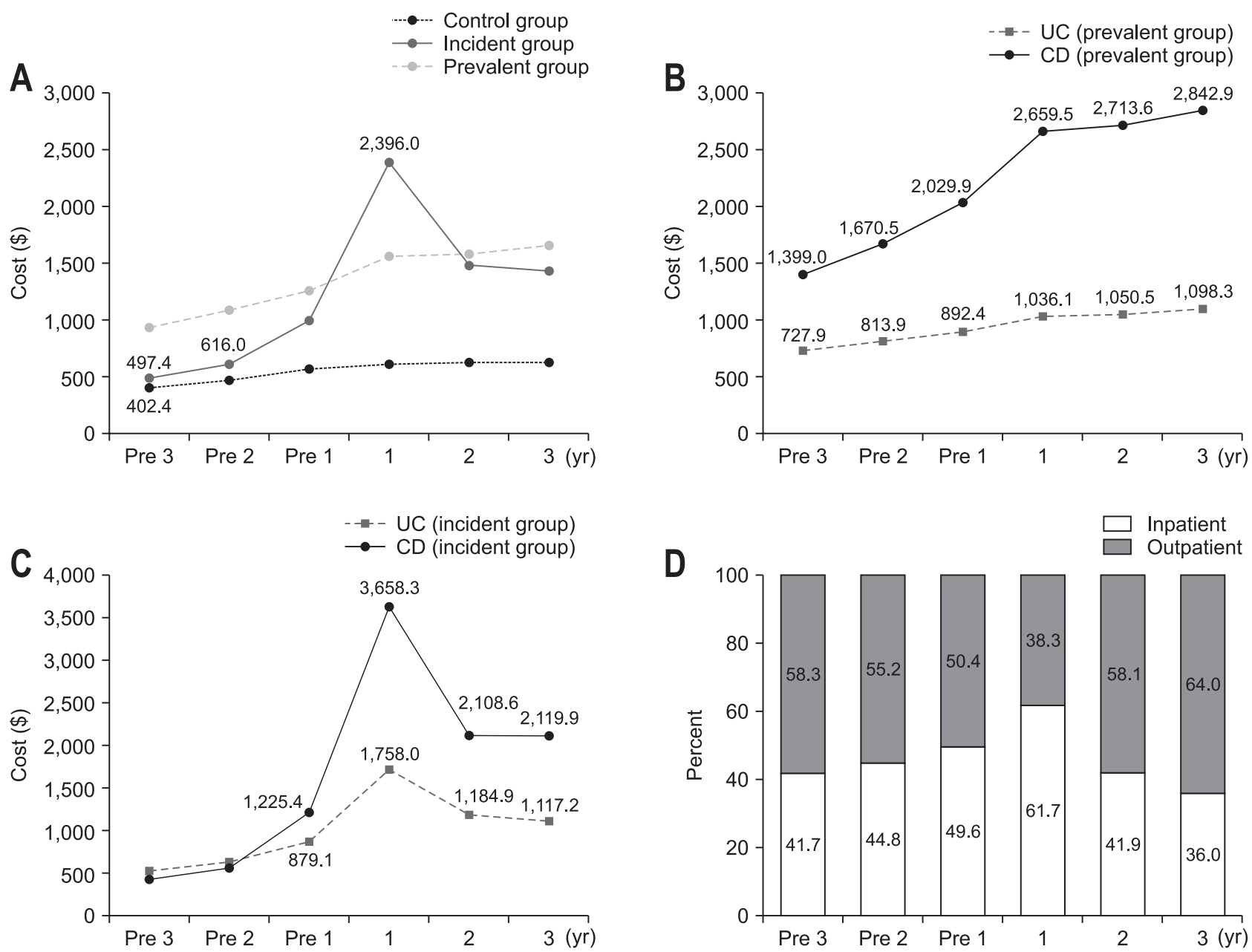

Fig. 1. Changes in the total healthcare cost of (A) the IBD group (the incident and the prevalent group) and the control group, (B) the prevalent group and (C) the incident group. (D) Proportions of inpatient costs and outpatient costs. IBD, inflammatory bowel disease; UC, ulcerative colitis; CD, Crohn's disease. 
$\$ 2,396$ during the first year after the IBD diagnosis. This cost sharply declined during the second year after diagnosis and was comparable to the cost in the prevalent group ( $\$ 1,478$ vs $\$ 1,579, p=0.002)$. Total healthcare costs per patient in the prevalent group gradually increased throughout the study period from $\$ 940$ to $\$ 1,654$ (Fig. 1A).

In the prevalent group, $\mathrm{CD}$ was associated with approximately 2-fold higher healthcare costs than UC throughout the study period (Fig. 1B). In the incident group, the costs for $\mathrm{CD}$ were consistently higher than those for UC from 1 year before diagnosis and onward (Fig. 1C). Inpatient costs of the incident group were the highest during the first year after diagnosis $(\$ 1,479)$ and subsequently decreased, with outpatient costs exceeding inpatient costs from the second year after diagnosis and onward. The proportion of outpatient costs gradually increased from $38 \%$ to $64.0 \%(\mathrm{p}<0.001)$ (Fig. 1D).

\section{Costs of diagnostic tests}

Figs 2 and 3 show the changes in the cost of endoscopy and radiologic imaging for patients before and after IBD diagnosis compared to that of the control group. The costs of endoscopy and radiologic imaging were higher for IBD patients (both of the incident group and the prevalent group) than for the control group throughout the study period (Figs 2A, 3A). The costs of endoscopy and radiologic imaging were also higher for the

A

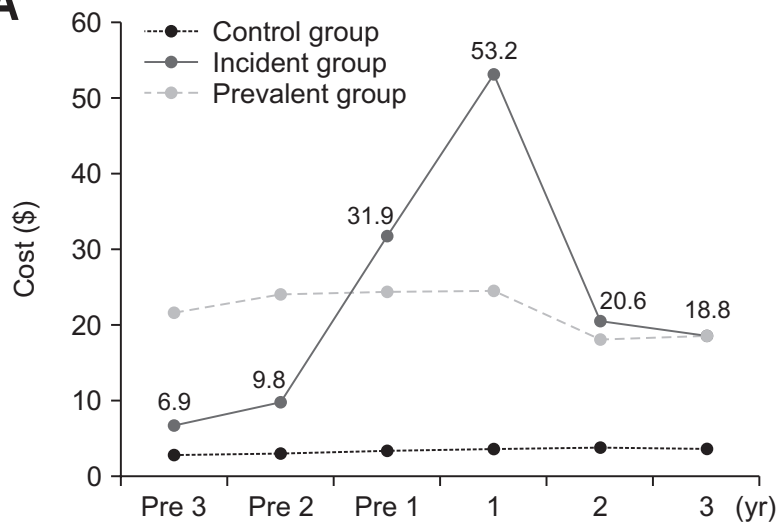

C

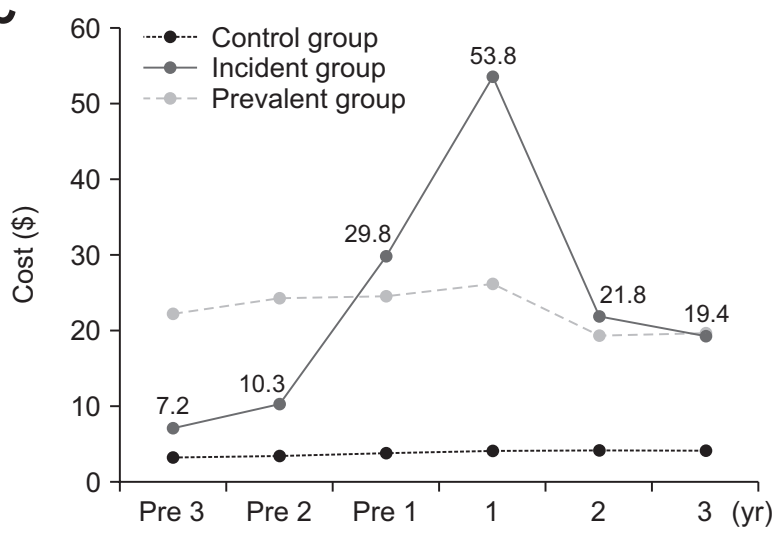

incident group than for the control group, even up to 3 years before the diagnosis. (\$6.9 vs $\$ 2.9$ and $\$ 4.2$ vs $\$ 2.7$, respectively, both $\mathrm{p}<0.001)$. The differences in costs between the incident group and the control group gradually increased until 1 year after the diagnosis. The cost of endoscopy in the incident group peaked at \$53.2 during the first year after diagnosis, but subsequently decreased and became comparable to the cost in prevalent group during the third year after diagnosis (\$18.8 vs $\$ 18.6, p=0.52$ ). A similar trend was also observed for radiologic imaging, which peaked during the first year after diagnosis (\$53.8) and then decreased to become comparable to the cost in the prevalent group during the third year after diagnosis (\$17.6 vs $\$ 18.9, p=0.14)$. This tendency was the same when total IBD was classified into CD and UC respectively, although the cost of radiologic imaging in $\mathrm{CD}$ patients was significantly higher than that in UC patients (Fig. 3B and C).

\section{Costs of surgery}

The changes in costs of surgery per patient of study population are depicted in Fig. 4. As mentioned above, the cost of surgery included the cost of all operations related to IBD, from minor surgery, including an abscess drainage, to major surgery, including bowel resection, and are expressed as mean cost per patient. Costs of surgery in IBD patients were the highest after 1 year of IBD diagnosis and decreased sharply from the second

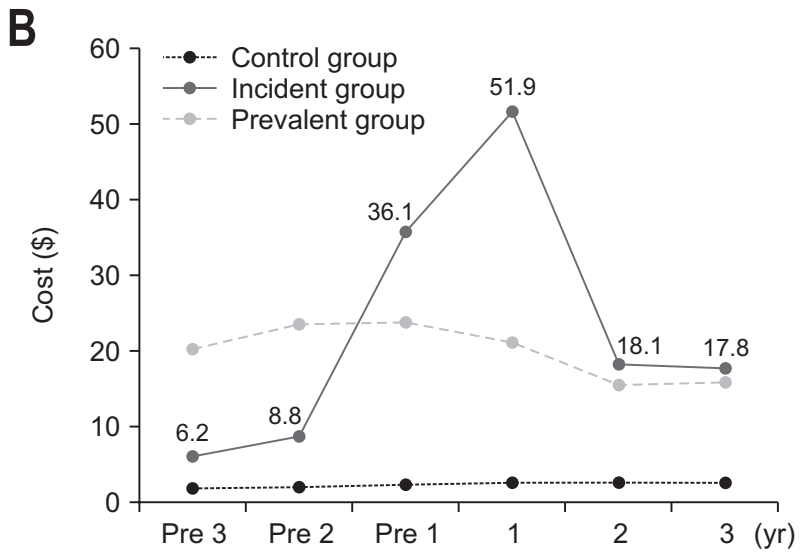

Fig. 2. Cost of endoscopy for the (A) total inflammatory bowel disease group (the incident and the prevalent group) and the control group, (B) Crohn's disease patients (the incident and the prevalent group) and the control group and (C) ulcerative colitis patients (the incident and the prevalent group) and the control group. 

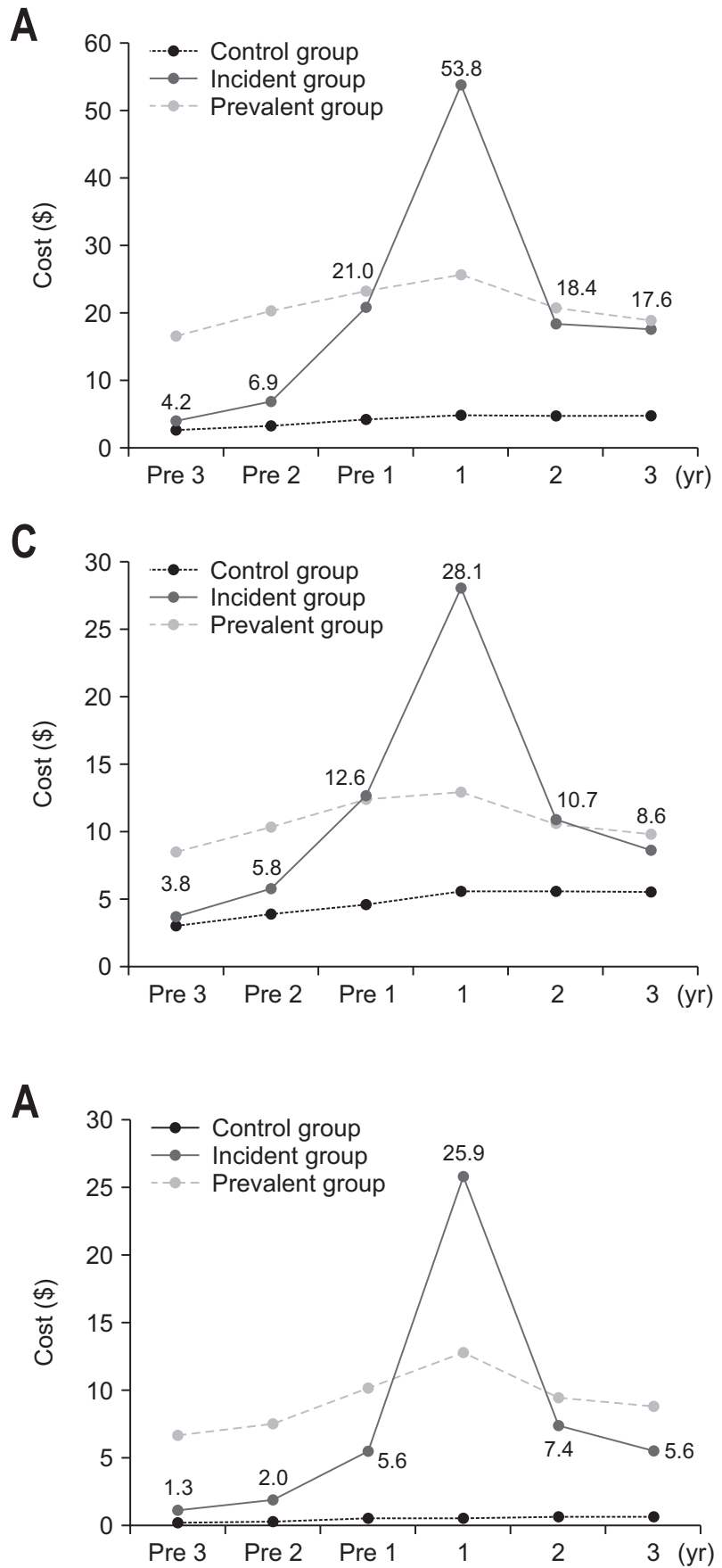

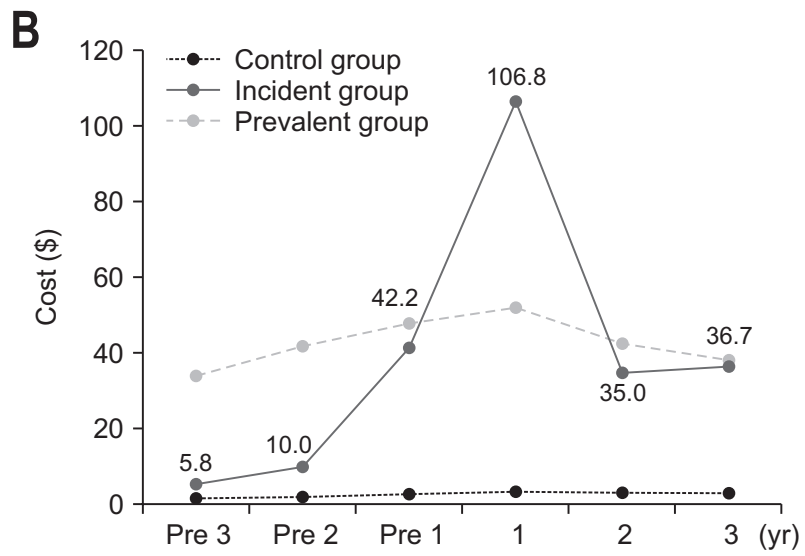

Fig. 3. Cost of radiologic imaging for the (A) total inflammatory bowel disease group (the incident and the prevalent group) and the control group, (B) Crohn's disease patients (the incident and the prevalent group) and the control group and (C) ulcerative colitis patients (the incident and the prevalent group) and the control group.

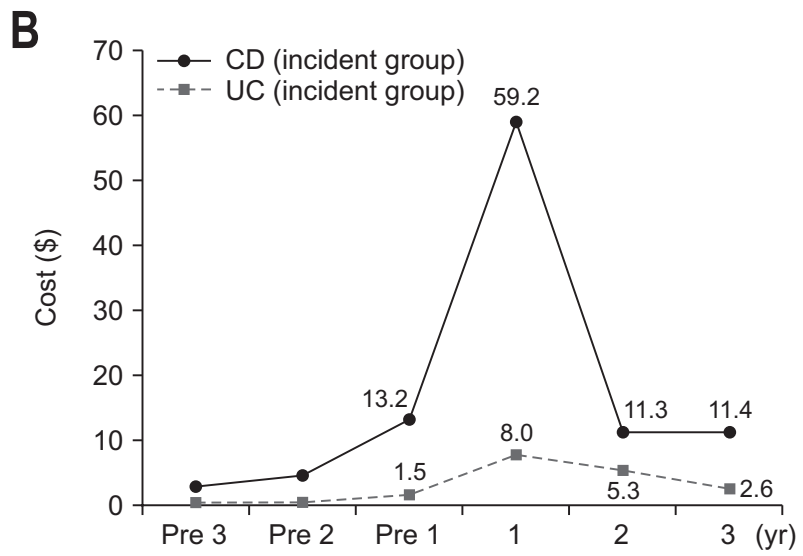

Fig. 4. Cost of surgery for the (A) total inflammatory bowel disease group (the incident and the prevalent group) and the control group and (B) Crohn's disease (CD) patients versus ulcerative colitis (UC) patients in the incident group.

year of diagnosis. In addition, for the second year of diagnosis, costs of surgery in the incident group were lower than those of the prevalent group, but the differences were not statistically significant (Fig. 4A). In the incident group, the cost of surgery for CD patients was 7-fold higher than that of UC patients, 1 year after diagnosis. And, even 2 years after the diagnosis, the costs of the CD patients were significantly higher than those of UC patients $(\mathrm{p}<0.05)$ (Fig. 4B).

\section{Costs of biologics}

The mean costs of biologics for IBD increased steeply over time, from $\$ 720.8$ during the first year after diagnosis to $\$ 1,249.6$ during the third year after diagnosis $(\mathrm{p}<0.001)$ (Table 2, Fig. 5A). This trend was common in both CD and UC, although CD was associated with higher biologics costs than UC. In the incident group, the cost of biologics treating CD patients was \$774 during the first year of after diagnosis, which was ap- 

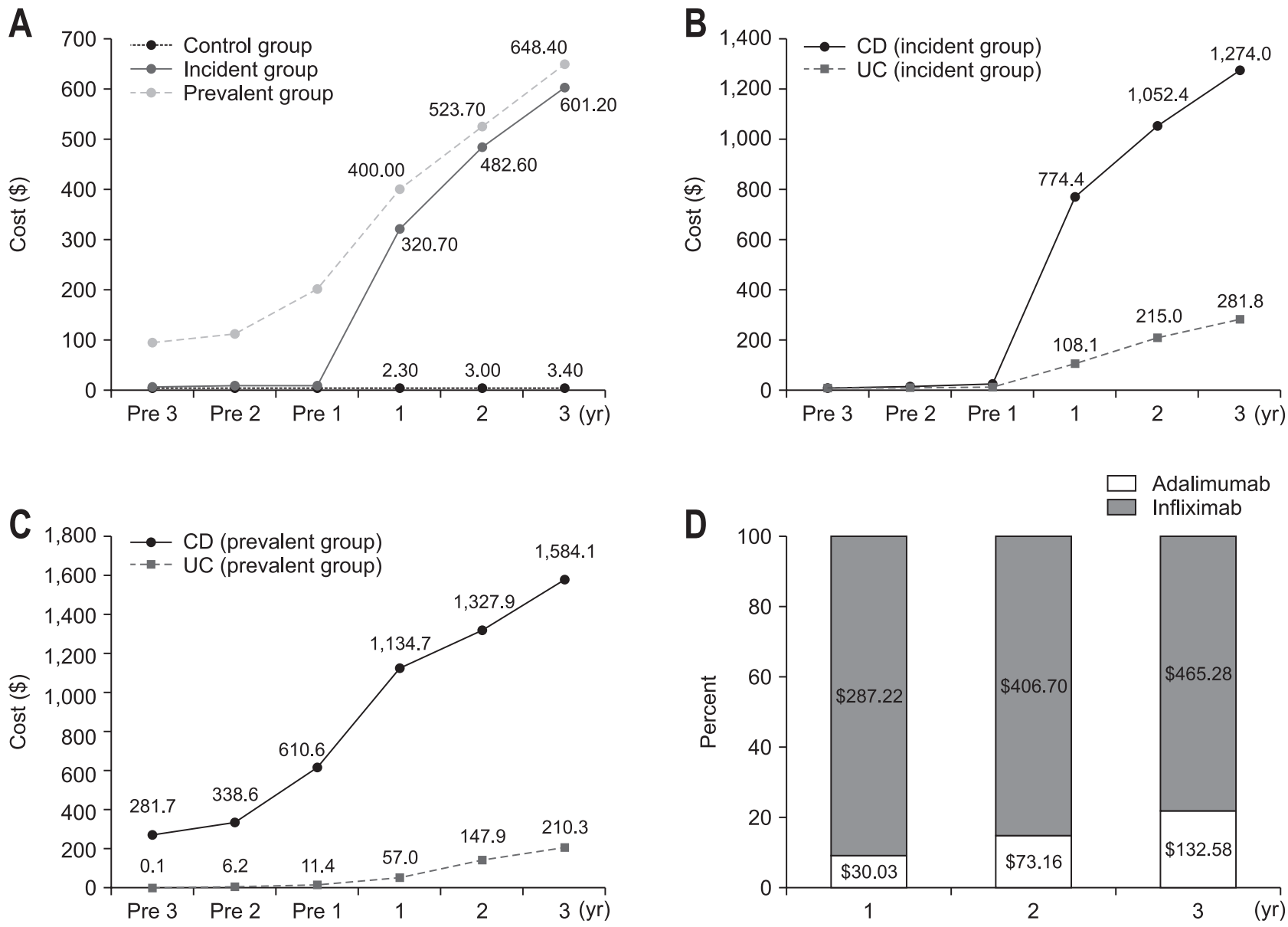

Fig. 5. Cost of biologics for the (A) total inflammatory bowel disease (IBD) group (the incident and the prevalent group) and the control group, (B) Crohn's disease (CD) patients versus ulcerative colitis (UC) patients in the incident group, and (C) CD patients versus UC patients in the prevalent group. (D) Changes in cost profiles of infliximab and adalimumab after IBD diagnosis.

proximately seven times the mean cost of $\$ 108$ for patients with UC (Fig. 5B). In the prevalent group, the cost of biologics for treating $\mathrm{CD}$ patients was $\$ 1,134.7$ during the first year after diagnosis, versus $\$ 57.0$ for treating UC patients during the same period (Fig. 5C). The mean per-patient costs for infliximab and adalimumab increased from \$287.2 and \$30.0 during the first year after diagnosis to $\$ 465.3$ and $\$ 132.6$ during the third year after diagnosis. The proportion of costs for infliximab decreased from $90.5 \%$ during the first year to $77.8 \%$ during the third year $(p<0.001)$, while the proportion for adalimumab increased from $9.4 \%$ during the first year to $22.2 \%$ during the third year ( $p<0.001)$ (Fig. 5D).

\section{DISCUSSION}

A recent population-based study in South Korea revealed that the steadily rising prevalence of IBD was associated with a dramatic increase in IBD-related healthcare costs from \$23.2 million during 2010 to $\$ 49.7$ million during 2014 . $^{18}$ Furthermore, the present study focused on the changes in direct healthcare costs per patient before and after the IBD diagnosis, and com- pared the costs for patients with and without IBD in a national claimed database.

We showed that patients newly diagnosed with IBD (the incident group) had the highest healthcare costs during the first year of diagnosis, and the costs subsequently decreased and became comparable to those of formerly diagnosed patient (the prevalent group) after 2 years of diagnosis. Based on this result, we cautiously assumed that the IBD-related direct healthcare costs are concentrated at the early stage of disease course and then relatively stabilize to some extent thereafter, as similar trends have been observed in previous studies. For example, the direct IBD-related healthcare costs in Hong Kong were higher in the first year after diagnosis than in the second year after diagnosis. ${ }^{19}$ In addition, an incidence-based cost analysis by the European Collaborative Study Group on Inflammatory Bowel Disease revealed that total and hospitalization-related costs were much higher in the first year of diagnosis than in subsequent years. ${ }^{15}$ This trend could be related to the increased need for diagnostic testing and aggressive treatment that aims to achieve remission during the first year after diagnosis. ${ }^{20}$

Interestingly, the incident group had higher overall healthcare 
costs than the control group, even before their IBD diagnosis, and this trend was observed in all categories of cost (inpatient, outpatient and diagnostic test). We suggest that these results could be related to diagnostic delay, which is observed for a considerable number of patients with IBD, as their symptoms are nonspecific and the available tests have limited diagnostic accuracy. A European study showed that approximately 20\% of the 4,670 patients were only diagnosed at $>5$ years after noticing their first symptoms, and $67 \%$ of the patients had visited an emergency department at least once before the diagnosis. ${ }^{21}$ Moreover, a Korean cohort study revealed that the mean diagnostic delay was $16.0 \pm 33.0$ months, ${ }^{22}$ which suggests that a timely diagnosis and treatment could reduce healthcare costs that are related to pre-diagnosis hospital visits or admission.

However, South Korea has lower diagnostic costs for endoscopy or radiologic imaging than in other Asian or Western countries. The cost of biologics was more than $\$ 700$ during the first year after diagnosis, which was seven times higher than the cost of diagnostic testing. Therefore, we can cautiously assume that biologics could be a main driver of costs during the early period after the IBD diagnosis. In both the incident group and the prevalent group, the costs of $\mathrm{CD}$ patients were higher than those of UC. The fact that biologics are used more as treatment in CD than in UC seems to be the cause of these cost differences.

Several previous studies have indicated that a top-down strategy using biologics for early-stage IBD provided greater effectiveness than a step-up strategy during clinical remission, ${ }^{23-26}$ although changes in the paradigm of IBD treatment may have led to increases in related healthcare costs. ${ }^{27}$ In South Korea, the NHI expanded coverage for adalimumab to adult patients with CD that was refractory to conventional medical treatment in 2010, while infliximab and adalimumab were approved for adults with refractory UC in 2011 and 2011, respectively. These changes have improved access to biologics for IBD and increased the use of adalimumab, with 306 patients receiving biologics in $2007(\$ 1,816,065)$ versus 5,223 patients receiving biologics in $2015(\$ 38,328,839)$ (Supplementary Table 1 , Supplementary Fig. 1). The importance of biologics will be more emphasized in the future and the introduction of new biologics is expected to increase. Therefore, establishment of the health insurance policy that reflects this trend are required and the introduction of biosimilar agents at a lower price than originator biologics may also help further reduce healthcare costs. In addition, considering the primary nonresponse to induction therapy with biologics, secondary loss of response, or drug-related adverse events, individualized therapy with tight monitoring and patient care by IBD specialist should be accompanied to increase cost-effectiveness of biologics use. An Asian study revealed that high cost (>90th percentile) outliers were associated with IBDrelated surgery. ${ }^{19}$ Our study showed that the costs of surgery were the highest in 1 year after diagnosis and CD was associated with higher costs of surgery. However, as its absolute cost was not high, it is unlikely to account for a substantial proportion of the total direct healthcare cost of IBD in South Korea. The likelihood of surgery increases over time based on the natural course of IBD and several studies have reported that the proportion of surgery-related healthcare costs for treating IBD has decreased during the 21st century. ${ }^{4,28-31}$

The present study has several limitations. First, data restrictions and limited accessibility to data precluded detailed analyses of costs for medications, such as 5-aminosalicylic acid or azathioprine, which are important drivers of costs in Western studies. We were able to analyze only biologics that were prescribed and paid in hospital and the billing code was collected in the NHI database. Oral agents are essential means of treating IBD, and further studies of healthcare costs including medication are warranted. Second, costs according to disease behavior or IBD severity were not evaluated because these data were not available from the NHI database. This should also be analyzed through further research.

Despite these limitations, this study is the first populationbased study to analyze the changes in costs including biologics before and after IBD diagnosis in East Asia, which has been driven by the increasing incidence and prevalence of IBD. As the use of biologics in IBD is expected to accelerate and several new agents being evaluated, these findings may be relevant for predicting future direct healthcare costs in Asian countries. In a survey of 599 patients with IBD, 41\% of respondents reported decreased quality of life and 21\% reported stopping medical treatment because of the related economic burden. ${ }^{32}$ As economic burden is closely associated with the outcomes of IBD management, cost-effective healthcare policies are needed to lessen the economic burden of this chronic disease.

In conclusion, patients with newly diagnosed IBD incurred the highest direct healthcare costs during the first year after diagnosis, and IBD patients had higher costs than individuals without IBD even before the diagnosis of IBD. In addition, the cost of biologics increased steeply over time and it can be assumed that biologics could be the main driver of costs during the early period after IBD diagnosis in South Korea.

\section{CONFLICTS OF INTEREST}

No potential conflict of interest relevant to this article was reported.

\section{ACKNOWLEDGEMENTS}

This work was supported by a grant of the Korean Association for the Study of Intestinal Disease for 2017.

\section{AUTHOR CONTRIBUTIONS}

Concept and design: J.L., J.P.I. Analysis and interpretation of 
the data: K.H., J.L., J.P.I. Drafting of the article: J.L., J.P.I. Critical revision of the article for important intellectual content: J.K., H.J.L., J.C., J.S.K. Final approval of the article: all authors.

\section{ORCID}

Jooyoung Lee Jong Pil Im

Kyungdo Han Jihye Kim

Hyun Jung Lee Jaeyoung Chun Joo Sung Kim https://orcid.org/0000-0003-1584-0160 https://orcid.org/0000-0002-6096-1263 https://orcid.org/0000-0003-0763-2935 https://orcid.org/0000-0003-2764-9339 https://orcid.org/0000-0002-4212-0380 https://orcid.org/0000-0001-6835-4735
https://orcid.org/0000-0003-1652-088X

\section{REFERENCES}

1. Gunnarsson C, Chen J, Rizzo JA, Ladapo JA, Lofland JH. Direct health care insurer and out-of-pocket expenditures of inflammatory bowel disease: evidence from a US national survey. Dig Dis Sci 2012;57:3080-3091.

2. Kappelman MD, Rifas-Shiman SL, Porter CQ, et al. Direct health care costs of Crohn's disease and ulcerative colitis in US children and adults. Gastroenterology 2008;135:1907-1913.

3. Rocchi A, Benchimol EI, Bernstein CN, et al. Inflammatory bowel disease: a Canadian burden of illness review. Can J Gastroenterol 2012;26:811-817.

4. van der Valk ME, Mangen MJ, Leenders M, et al. Healthcare costs of inflammatory bowel disease have shifted from hospitalisation and surgery towards anti-TNFalpha therapy: results from the COIN study. Gut 2014;63:72-79.

5. Ng SC. Emerging leadership lecture: inflammatory bowel disease in Asia: emergence of a "Western" disease. J Gastroenterol Hepatol 2015;30:440-445.

6. Singh P, Ananthakrishnan A, Ahuja V. Pivot to Asia: inflammatory bowel disease burden. Intest Res 2017;15:138-141.

7. Yang SK, Yun S, Kim JH, et al. Epidemiology of inflammatory bowel disease in the Songpa-Kangdong district, Seoul, Korea, 1986-2005: a KASID study. Inflamm Bowel Dis 2008;14:542-549.

8. Kim HJ, Hann HJ, Hong SN, et al. Incidence and natural course of inflammatory bowel disease in Korea, 2006-2012: a nationwide population-based study. Inflamm Bowel Dis 2015;21:623-630.

9. Ng WK, Wong SH, Ng SC. Changing epidemiological trends of inflammatory bowel disease in Asia. Intest Res 2016;14:111-119.

10. Jung YS, Han M, Kim WH, Park S, Cheon JH. Incidence and clinical outcomes of inflammatory bowel disease in South Korea, 2011-2014: a nationwide population-based study. Dig Dis Sci 2017;62:2102-2112.

11. Lee YH, Han K, Ko SH, Ko KS, Lee KU; Taskforce Team of Diabetes Fact Sheet of the Korean Diabetes Association. Data analytic process of a nationwide population-based study using National Health Information Database established by National Health Insurance Service. Diabetes Metab J 2016;40:79-82.
12. Kim ES, Jeong JS, Han K, et al. Impact of weight changes on the incidence of diabetes mellitus: a Korean nationwide cohort study. Sci Rep 2018;8:3735.

13. Park S, Chun J, Han KD, et al. Increased end-stage renal disease risk in patients with inflammatory bowel disease: a nationwide population-based study. World J Gastroenterol 2018;24:47984808.

14. Soh H, Chun J, Han K, et al. Increased risk of herpes zoster in young and metabolically healthy patients with inflammatory bowel disease: a nationwide population-based study. Gut Liver 2019;13:333-341.

15. Odes S, Vardi H, Friger M, et al. Cost analysis and cost determinants in a European inflammatory bowel disease inception cohort with 10 years of follow-up evaluation. Gastroenterology 2006;131:719-728.

16. Drummond M. Methods for the economic evaluation of health care programmes. 4th ed. Oxford: Oxford University Press, 2015.

17. Niewiadomski O, Studd C, Hair C, et al. Health care cost analysis in a population-based inception cohort of inflammatory bowel disease patients in the first year of diagnosis. J Crohns Colitis 2015;9:988-996.

18. Kim JW, Lee CK, Rhee SY, Oh CH, Shim JJ, Kim HJ. Trends in health-care costs and utilization for inflammatory bowel disease from 2010 to 2014 in Korea: a nationwide population-based study. J Gastroenterol Hepatol 2018;33:847-854.

19. Mak LY, Ng SC, Wong IOL, et al. Direct health-care cost utilization in Hong Kong inflammatory bowel disease patients in the initial 2 years following diagnosis. J Gastroenterol Hepatol 2018;33:141149.

20. Petryszyn PW, Witczak I. Costs in inflammatory bowel diseases. Prz Gastroenterol 2016;11:6-13.

21. Lönnfors S, Vermeire S, Greco M, Hommes D, Bell C, Avedano L. IBD and health-related quality of life: discovering the true impact. J Crohns Colitis 2014;8:1281-1286.

22. Moon CM, Jung SA, Kim SE, et al. Clinical factors and disease course related to diagnostic delay in Korean Crohn's disease patients: results from the CONNECT Study. PLoS One 2015;10:e0144390.

23. D'Haens G, Baert F, van Assche G, et al. Early combined immunosuppression or conventional management in patients with newly diagnosed Crohn's disease: an open randomized trial. Lancet 2008;371:660-667.

24. Schreiber S, Colombel JF, Bloomfield R, et al. Increased response and remission rates in short-duration Crohn's disease with subcutaneous certolizumab pegol: an analysis of PRECiSE 2 randomized maintenance trial data. Am J Gastroenterol 2010;105:1574-1582.

25. Colombel JF, Rutgeerts P, Reinisch W, et al. Early mucosal healing with infliximab is associated with improved long-term clinical outcomes in ulcerative colitis. Gastroenterology 2011;141:11941201.

26. Colombel JF, Reinisch W, Mantzaris GJ, et al. Randomised clinical trial: deep remission in biologic and immunomodulator naïve 
patients with Crohn's disease: a SONIC post hoc analysis. Aliment Pharmacol Ther 2015;41:734-746.

27. Im JP, Ye BD, Kim YS, Kim JS. Changing treatment paradigms for the management of inflammatory bowel disease. Korean J Intern Med 2018;33:28-35.

28. Frolkis AD, Dykeman J, Negrón ME, et al. Risk of surgery for inflammatory bowel diseases has decreased over time: a systematic review and meta-analysis of population-based studies. Gastroenterology 2013;145:996-1006.

29. Rungoe C, Langholz E, Andersson M, et al. Changes in medical treatment and surgery rates in inflammatory bowel disease: a nationwide cohort study 1979-2011. Gut 2014;63:1607-1616.

30. Ferrari L, Krane MK, Fichera A. Inflammatory bowel disease surgery in the biologic era. World J Gastrointest Surg 2016;8:363370.

31. Katsanos KH, Papadakis KA. Inflammatory bowel disease: updates on molecular targets for biologics. Gut Liver 2017;11:455-463.

32. Kim YS, Jung SA, Lee KM, et al. Impact of inflammatory bowel disease on daily life: an online survey by the Korean Association for the Study of Intestinal Diseases. Intest Res 2017;15:338-344. 